\title{
Need to use Fog Computing with IoT
}

\section{Tajinder Kaur}

P. G. Department of Computer Science, Sri Guru Teg Bahadur Khalsa College, Sri Anandpur Sahib, Punjab, India

Article Info

Volume 7, Issue 3

Page Number: 596-601

Publication Issue :

May-June-2021

\section{Article History}

Accepted : 18 June 2021

Published : 25 June 2021

\section{ABSTRACT}

The field of computing has covered a long path. From Mainframes to traditional desktop and then towards Ubiquitous Computing. Ubiquitous Computing combined with wireless Network embedded with cloud computing have given rise to new type of concept known as Internet of Things. But this is not the final destination. A new edge is added further to this which is known as Edge Computing or Fog Computing. With less maintenance and cost based on service usage, cloud computing's layered architecture allows the client to purchase services at different levels such as IaaS, PaaS and SaaS. It permits location independence facility as users can access these services anywhere with an internet connection and a web browser. IoT devices generate data constantly, and often analysis must be very rapid. Main sources of this huge amount of data are devices, sensors or actuators. Handling the volume, variety, and velocity of IoT data requires a new computing model. Also the time it takes to makes its way to the cloud for analysis, the opportunity to act on it might be gone. So, a new model which will further enhance the capabilities of IoT is explained. This new model is known as Fog Computing or Edge Computing. There are number of applications where this model fits. In this paper introduction to Iot and its applications are discussed. Then Fog computing is introduced.

Keywords : Internet of Things, IoT, Cloud Computing, Edge Computing, Fog Computing, RFID

\section{INTRODUCTION}

Several years ago a novel paradigm named "The Internet of Things (IoT)" has been introduced. Although this term was introduced by Kevin Ashton in 1999[1] which have increased the importance of it rigorously. Introducing IoT into embedded mobile transceivers into a wide range of devices, would add a whole new level to the world of information and communication technology.
The IoT aims to unify everything in our world under a common infrastructure, giving us not only control of things around us, but also keeping us informed of the state of the things.

In the Internet of Things (IoT) paradigm, 'things' can be any object or a person and all the things will be on the network in one form or another.

It has the potential to not only affect the health, safety and productivity of billions of people but also has a major economic impact. It primarily consists of physical objects that are embedded with sensors,

Copyright: @ the author(s), publisher and licensee Technoscience Academy. This is an open-access article distributed under the terms of the Creative Commons Attribution Non-Commercial License, which permits unrestricted non-commercial use, distribution, and reproduction in any medium, provided the original work is properly cited 
actuators, computing devices and data communication capabilities. These are linked to networks for data transportation.

The idea of IoT has been scrutinized in the 20th Tyrrhenian workshop on digital communications. IoT is described as a connection of various "things" or "objects" around us, like, sensors, mobile phones, Radio Frequency Identification (RFID tags) which work through a unique addressing system with which these things are able to communicate with each other and complete their tasks successfully [2]. IoT mainly depends on Wireless Sensor Networks (which compile the information collection, processing and transformation), intelligent technologies (in order to solve problems and initialize and control the machine-to-machine interaction) and Nano-meter technologies (to construct small devices in order to socialize the IOT applications). There is still research being conducted in the field of IoT in order to make it more easy to use and also increase the security of the information transferred through it. The best architecture is the Unique Identifier (UID) in japan and the EPC global networks supported by Auto-Id.

Smart connectivity with existing networks and context-aware computation using network resources is an indispensable part of IoT. With the growing presence of WiFi and LTE wireless Internet access, the evolution towards ubiquitous information and communication networks is already evident. However, for the Internet of Things vision to successfully emerge, the computing paradigm will need to go beyond traditional mobile computing scenarios that use smart phones and portables, and evolve into connecting everyday existing objects and embedding intelligence into our environment [3].

\section{Applications of IoT in various areas}

The key feature in IoT is, without doubt, its impact on every-day life of potential users. IoT has remarkable effects both in work and home scenarios, where it can play a leading role in the next future (assisted living, domestics, e-health, smart transportation, etc.)[4].

- e-health :An extension of the personal body area network is creating a home monitoring system for aged-care, which allows the doctor to monitor patients and elderly in their homes thereby reducing hospitalization costs through early intervention and treatment [5].

- Smart Home: Control of home equipment such as air conditioners, refrigerators, washing machines etc., will allow better home and energy management. This will see consumers become involved in the IoT revolution in the same manner as the Internet revolution itself [6].

- Smart transportation :The transport IoT will enable the use of large scale WSNs for online monitoring of travel times, origin-destination (OD) route choice behavior, queue lengths and air pollutant and noise emissions.

- Smart Logistics: Efficient logistics management includes monitoring the items being transported as well as efficient transportation planning [7].

- Smart Transportation: The transport IoT will enable the use of large scale WSNs for online monitoring of travel times, origin-destination (OD) route choice behavior, queue lengths and air pollutant and noise emissions [7].

- Smart Environment IoT: The applications or usecases within the urban environment includes the effect on citizens considering health and wellbeing issues; transport in light of its impact on mobility, productivity, pollution; and services in terms of critical community 


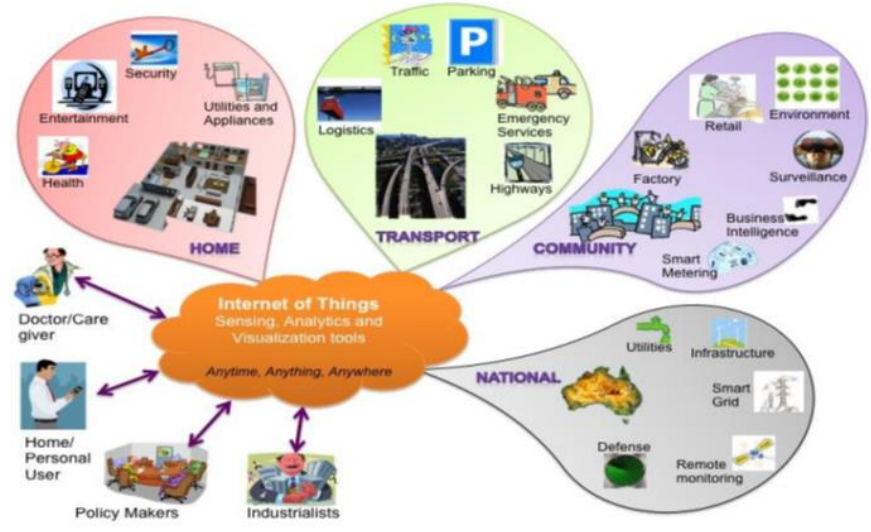

Figure 1: Internet of Things Schematic showing the end users and application areas based on data services managed and provided by local government to city inhabitants [8]

- Smart grid and smart metering: This is another potential IoT application. Efficient energy consumption can be achieved by continuously monitoring every electricity point within a house and using this information to modify the way electricity is consumed. This information at the city scale is used for maintaining the load balance within the grid ensuring high quality of service[9].

- Video based IoT : It integrates image processing, computer vision and networking frameworks, will help develop a new challenging scientific research area at the intersection of video, infrared, microphone and network technologies.

- Water network monitoring and quality assurance of drinking water: This is another critical application that is being addressed using IoT. Sensors measuring critical water parameters are installed at important locations in order to ensure high supply quality.

A schematic of the interconnection of objects is depicted in Figure 1, where the application domains are chosen based on the scale of the impact of the data generated. The users span from an individual to national level organizations addressing wide ranging issues.

\section{Challenges faced in actual implementation of IoT}

There are challenges and issues which need to be addressed for the proper implementation of IoT[10]:

Addressing schemes: The ability to uniquely identify 'Things' is critical for the success of IoT. This will not only allow us to uniquely identify billions of devices but also to control remote devices through the Internet. The few most critical features of creating a unique address are: uniqueness, reliability, persistence and scalability. So this is an important issue for research.

Interoperability issues: An emerging problem today is that there are still many vendors who do not support certain approved standards in their products, thus leading to significant interoperability issues and increased system integration costs.

Different Syntax and structure of data from various data sources: Device interfacing and data collection will not yield results unless the structure and syntax of data and its meaning is properly understood. Intelligent applications or mashups can be built only when the semantics is understood. When their semantics are clear then different techniques such as correlation, complex event processing and automated reasoning with semantics technology can be applied. So the semantics of the data must be part of the data itself and should not be locked up within the application logic in different applications.

Big Data Problem: The amount of data that needs to be ingested, stored and analyzed will also increase exponentially which will lead to a typical 'Big Data' problem where the sheer volume and velocity of data received will make standard architectures and platforms inadequate.

Real time performance required: Some applications may demand high real-time performance than what is normally possible using standard internet technologies. 


\section{Integration of Cloud Computing and IoT}

For the realization of a complete IoT vision, an efficient, secure, scalable and market oriented computing and storage resourcing is essential. Cloud computing is the most recent paradigm to emerge which promises reliable services delivered through next generation data centres that are based on virtualised storage technologies. This platform acts as a receiver of data from the ubiquitous sensors; as a computer to analyze and interpret the data; as well as providing the user with easy to understand web based visualization. The ubiquitous sensing and processing works in the background, hidden from the user.

In general, IoT can take advantage from the virtually unlimited capabilities and resources of Cloud to meet its technological constraints like storage, processing and communication.

Cloud computing can provide the virtual infrastructure for such utility computing which integrates monitoring devices, storage devices, analytics tools, visualization platforms and client delivery. Cloud computing's layered architecture allows the client to purchase services at different levels such as IaaS, PaaS and SaaS. The cost based model that Cloud computing offers will enable end-to-end service provisioning for businesses and users to access applications on demand from anywhere.

Most of the papers in literature believe that Cloud fills some gaps of IoT like the limited storage capability of IoT whereas a few others believe that IoT fills gaps of Cloud for example the limited scope of Cloud can be extended to deal with real world things in a more distributed and dynamic manner[11].

Although Cloud can significantly improve and simplify IoT communication, it can still represent a bottleneck in some scenarios. For example: practical limitations can arise when trying to transfer huge amounts of data from the edge of the Internet onto Cloud as data storage density and processor power increased of a factor of $10^{18}$ and $10^{15}$ respectively, while broadband capacity increased only of $10^{4}$.

\section{Limitations of Cloud Computing}

Cloud provides new opportunities in aggregating sensor data and exploiting the aggregates for larger coverage and relevancy, but at the same time affects privacy and security.

CloudIoT inherits the same monitoring requirements from Cloud, but the related challenges are further affected by volume, variety, and velocity characteristics of IoT.

Latency-sensitive applications cannot depend upon cloud computing services to take its decisions. There is a requirement of a system which can handle the data coming from many sources and can take quick actions with the help of artificial intelligence.

\section{Introduction to Fog Computing}

The Internet of Things (IoT) is generating an unprecedented volume and variety of data. But by the time the data makes its way to the cloud for analysis, the opportunity to act on it might be gone. So a new model for analyzing and acting on IoT data has been introduced. This is known as either edge computing or Fog computing [12].

- Analyzes the most time-sensitive data at the network edge, close to where it is generated instead of sending vast amounts of IoT data to the cloud.

- Acts on IoT data in milliseconds, based on policy.

- Sends selected data to the cloud for historical analysis and longer-term storage.

It acts as an intermediator between huge data and cloud computing. Fog computing is an extension of classic Cloud computing to the edge of the network. It serves the purpose by handling huge amount of data at localised servers and then with help of artificial intelligence at specific nodes, quick decisions could be taken and actions can be performed with the help of actuators. 
Its impact on real time problems: Critical medical situations can be benefitted where 24 hour surveillance is needed from the doctor.

Fog: The fog extends the cloud to be closer to the things that produce and act on IoT data. These devices, called fog nodes, can be deployed anywhere with a network connection: on a factory floor, on top of a power pole, alongside a railway track, in a vehicle, or on an oil rig. Any device with computing, storage, and network connectivity can be a fog node. Examples include industrial controllers, switches, routers, embedded servers, and video surveillance cameras.

\section{Fog and Cloud together}

Fog Computing is, essentially, a middle layer between the cloud and the hardware to enable more efficient data processing, analysis and storage, which is achieved by reducing the amount of data which needs to be transported to the cloud.

While Fog nodes provide localization, therefore enabling low latency and context awareness, the Cloud provides global centralization. Many applications require both Fog localization, and Cloud globalization, particularly for analytics and Big Data[13].

In general, the Fog nodes and cloud platform will perform the following tasks:

\section{Fog nodes:}

- Some protocol will be used to receive feeds from IoT devices in real time

- Run specific IoT-enabled applications for realtime control and analytics, with millisecond response time.

- Provide short storage of 1 or 2 hours

- Periodic data summaries will be sent to the cloud for further processing.

\section{The cloud platform}

- Data summaries from various fog nodes will be received and aggregated.

- Then it will perform analysis on the IoT data and data from other sources to gain business insight.

- Updated application rules can be sent to the fog nodes based on these insights.

\section{Future scope}

Building Fog computing projects is a challenging task. Because the adoption of Fog-based approaches requires various algorithms and methodologies that can deal with reliability of the networks of smart devices, and their operation under specific conditions that ask for fault tolerant techniques.

So proper standards, rules and architecture need to be explored for the proper integration of IoT devices, Wireless sensor network, Fog nodes, Artificial intelligence, Internet, Cloud Platform and Big Data.

\section{IX.Conclusion}

In this paper, a new concept 'Internet of Things' is reviewed. A brief overview of its application in real life is given. Then the challenges faced in actual implementation of IoT were discussed. Then some reasons were outlined as to why various researchers are trying to integrate IoT and Cloud to give CloudIoT Paradigm. Then limitations of simply using cloud with IoT were highlighted. To remove these limitations a new approach need to be merged. This new model is known as Fog Computing.

Fog computing accelerates awareness and response to events by eliminating a round trip to the cloud for analysis. So, it avoids the need for costly bandwidth additions by offloading gigabytes of network traffic from the core network. It also protects sensitive IoT data by analyzing it inside organisation walls. 
A lot of work needs to be done in future for successful implementation of IoT, Fog and Cloud Computing collaboratively.

\section{REFERENCES}

[1]. K. Ashton, That Internet of Things, RFiD Journal. (2009).

[2]. H. Sundmaeker, P. Guillemin, P. Friess, S. Woelfflé, Vision and challenges for realising the Internet of Things, Cluster of European Research Projects on the Internet of Things CERP IoT, 2010.

[3]. J.Gubbi, R. Buyya, S. Marusic, M. Palaniswamia, Internet of Things (IoT): A Vision, Architectural Elements, and Future Directions, Future Generation Computer Systems 29(7),July 2012.

[4]. G. Santucci, From Internet of Data to Internet of Things, Paper for the International Conference on Future Trends of the Internet, 2009.

[5]. L. Haiyan, C. Song, W. Dalei, N. Stergiou, S. Ka-Chun, A remote marker less human gait tracking for e-healthcare based on contentaware wireless multimedia communications, IEEE Wireless Communication 17 (2010) 4450.

[6]. M. Darianian, M.P. Michael, Smart Home Mobile RFID-based Internet-Of-Things Systems and Services, 2008 International Conference on Advanced Computer Theory and Engineering. (2008) 116-120.

[7]. H. Lin, R. Zito, M. Taylor, A review of traveltime prediction in transport and logistics, Proceedings of the Eastern Asia Society for Transportation Studies. 5 (2005) 1433-1448.

[8]. R. Johnstone, D. Caputo, U. Cella, A. Gandelli, C. Alippi, F. Grimaccia, et al., Smart Environmental Measurement \& Analysis Technologies (SEMAT): Wireless sensor networks in the marine environment, in: Stockholm, 2008.

[9]. M. Yun, B. Yuxin, Research on the architecture and key technology of Internet of Things (IoT) applied on smart grid, Advances in Energy Engineering (ICAEE). (2010) 69-72.

[10]. Ahmed Banafa, IoT Standardization and Implementation Challenges, Newsletter, The IEEE Internet of Things, July 12, 2016.

[11]. A. Botta, W. d. Donato, V. Persico, A. Pescape,"Integration of Cloud computing and Internet of Things: A survey", Future Generation Computer Systems, Volume 56, March 2016, Pages 684-700.

[12]. Cisco- White Paper,Fog Computing and the Internet of Things: Extend the Cloud to Where the Things Are:

[13]. https://www.cisco.com/c/dam/en_us/solutions/t rends/iot/docs/computing-overview.pdf

[14]. F. Bonomi, R. Milito, J. Zhu, and S. Addepalli, "Fog computing and its role in the internet of things," Proceedings of the First Edition of the MCC Workshop on Mobile Cloud Computing, ser. MCC'12. ACM (2012), pp. 13-16.

\section{Cite this article as :}

Tajinder Kaur, "Need to use Fog Computing with IoT", International Journal of Scientific Research in Computer Science, Engineering and Information Technology (IJSRCSEIT), ISSN : 2456-3307, Volume 7 Issue 3, pp. 596-601, May-June 2021. Available at doi : https://doi.org/10.32628/CSEIT12173171

Journal URL : https://ijsrcseit.com/CSEIT12173171 